\title{
OCT4 regulates epithelial-mesenchymal transition and its knockdown inhibits colorectal cancer cell migration and invasion
}

\author{
XINZHENG DAI $^{1 *}$, JING GE $^{2 *}$, XUEHAO WANG $^{1}$, XIAOFENG QIAN ${ }^{1}$, \\ CHUANYONG ZHANG ${ }^{1}$ and XIANGCHENG LI ${ }^{1}$
}

\author{
${ }^{1}$ Liver Transplantation Center, First Affiliated Hospital of Nanjing Medical University, \\ Key Laboratory of Living Donor Liver Transplantation, Ministry of Public Health, Nanjing 210029; \\ ${ }^{2}$ Department of Endocrinology of Jiangsu Provincial Hospital of Traditional Chinese Medicine \\ Affiliated to Nanjing University of Traditional Chinese Medicine, Nanjing 210022, Jiangsu, P.R. China
}

Received August 31, 2012; Accepted September 24, 2012

DOI: $10.3892 /$ or.2012.2086

\begin{abstract}
Octamer-binding transcription factor 4 (OCT4) has been implicated in cancer metastasis. In this study, we investigated whether OCT4 promotes colorectal cancer (CRC) metastasis through the epithelial-mesenchymal transition (EMT) process. We designed our experiment as a loss-offunction study. Western blot analysis was used to measure the extent and stability of OCT4 knockdown. We evaluated the metastatic phenotype of OCT4-silenced SW620 cells using standard migration and invasion assays in vitro and the commonly used mouse model for experimental metastases in vivo. We found that OCT4 knockdown inhibited colorectal cancer cell motility and invasion (in vitro) and decreased hepatic colonization (in vivo). It also induced changes in EMT characteristic cell morphology and marker gene expression. In addition, its knockdown decreased WNT pathway activity. Finally, in human primary colorectal cancers, the frequency of upregulated OCT4 expression in cases with liver metastasis was statistically higher than that in cases without liver metastasis. These results indicate that OCT4 may contribute to CRC cell metastasis through EMT and serves as a promising
\end{abstract}

Correspondence to: Dr Xiangcheng Li or Dr Chuanyong Zhang, Liver Transplantation Center, First Affiliated Hospital of Nanjing Medical University, Key Laboratory of Living Donor Liver Transplantation, Ministry of Public Health, Guanzhou Road 300, Nanjing 210029, P.R. China

E-mail: xiangchenglinj@gmail.com

E-mail: chuanyong@yahoo.com

*Contributed equally

Abbreviations: OCT4, octamer-binding transcription factor 4; CRC, colorectal cancer; EMT, epithelial-mesenchymal transition

Key words: colorectal cancer, octamer-binding transcription factor 4, epithelial-mesenchymal transition, metastasis biomarker for identifying CRC patients at high risk for liver metastases.

\section{Introduction}

Colorectal cancer (CRC) is the third most common malignant neoplasm worldwide (1) and the second leading cause of death due to cancer (2). To date, the prognosis of CRC patients with liver metastasis remains poor. Therefore, it is necessary to clarify the molecular mechanisms involved in metastasis and to identify the specific biomarkers for predicting CRC liver metastasis.

Octamer-binding transcription factor 4 (OCT4), one of the key genes that induces pluripotent stem cells $(3,4)$, has also been implicated in several types of cancers including gastric cancer (5-7), breast cancer (8), non-small cell lung carcinomas (9), glioma (10-12) and esophageal squamous cell carcinoma (13). In addition, Saigusa et al (14) showed that OCT4 expression is associated with the distant recurrence of rectal cancer after chemoradiotherapy. Chang et al (15) demonstrated that OCT4 promoted tumorigenesis of CRC cells in both autocrine and paracrine manners. Gazouli et al (16) found that OCT4 expression levels were higher in CRC tissues compared to adjacent non-cancerous tissues, and the OCT4 expression levels in CRC tissues were correlated with tumor stage. Yet, to date the role of OCT4 in CRC metastasis and the potential molecular basis is unclear.

Epithelial-mesenchymal transition (EMT) is a wellcoordinated process that occurs during the progression of cancers and is necessary for metastasis of epithelial cancer (17-19). CRC cells at the invasive front acquire mesenchymal properties such as high migratory potential, poor differentiation, hyperproliferation and loss of cell-cell contact-mediated growth inhibition (18). In this study, we investigated whether OCT4 promotes CRC metastasis through the EMT process.

RNA interference is a powerful method for the research of gene function. We applied lentivirus-mediated shRNA to produce specific and stable silencing of OCT4 in SW620 CRC cells. We designed our experiment as a loss-of-function study. 
Western blot analysis was used to measure the extent and stability of OCT4 knockdown. We evaluated the metastatic phenotype of OCT4-silenced SW620 cells using standard migration and invasion assays in vitro and the commonly used mouse model for experimental metastases in vivo. The results revealed that OCT4 enhanced CRC cell metastasis by improving tumor cell invasion and migration. We further demonstrated that OCT4 knockdown in CRC SW620 cells induced MET (the reverse process of EMT) with characteristic morphological changes and changes in expression of EMT-associated key genes such as E-cadherin and vimentin. In addition, activity of the WNT pathway was significantly inhibited by OCT4 silencing in CRC SW620 cells. Finally, we showed that expression of OCT4 was correlated with CRC liver metastasis in human CRC tissues. Our study showed a novel role of OCT4 in regulating colorectal cancer metastasis by the EMT process, and OCT4 expression was identified as a promising biomarker for identifying colorectal cancer patients at high risk for liver metastasis.

\section{Materials and methods}

Cell culture. The human colorectal cell line SW620 was obtained from American Type Culture Collection (Manassas, VA, USA) and maintained in RPMI-1640 medium supplemented with $10 \%$ fetal bovine serum at $37^{\circ} \mathrm{C}$ in a $5 \% \mathrm{CO}_{2}$ atmosphere at constant humidity. The stable silencing of OCT4 in SW620 cells was generated by transduction with home made lentiviral particles for human OCT4 gene silencing.

Immunofluorescence cell staining. Cells were seeded on coverslips, incubated with primary antibody overnight at $4{ }^{\circ} \mathrm{C}$, washed with PBS and then incubated with secondary antibody conjugated with FITC (green) or Cy3 (red) (Millipore, USA) for $1 \mathrm{~h}$. Cells were imaged using a confocal microscope (Leica, Germany). Primary antibodies, anti-vimentin, anti-E-cadherin and anti- $\beta$-catenin, were purchased from Abcam (Cambridge, MA, USA).

Western blot analysis. Cells were lysed in RIPA cell lysis buffer (25 mM Tris-HCl, pH 7.6, $150 \mathrm{mM} \mathrm{NaCl,} \mathrm{1 \%} \mathrm{NP-40,}$ $1 \%$ sodium deoxycholate, $0.1 \%$ SDS) containing Protease Inhibitor Cocktail kit (Pierce, Rockford, IL, USA). Twenty micrograms of total cellular protein was loaded per lane, separated by $4-12 \%$ SDS-polyacrylamide gel electrophoresis and then transferred to nitrocellulose (Invitrogen, Carlsbad, CA, USA) by electroblotting (20). The antibodies anti-E-cadherin, anti-GAPDH, anti- $\beta$-catenin (1247-1, Epitomics), anti- $\alpha$-tublin and anti-TCF/LEF1 were purchased from Abcam.

$R N A$ isolation and RT-PCR. Total RNA was extracted from cells and then reverse transcribed. Quantitative real-time PCR reaction was performed in Biosystems 7500 (ABI, USA). All samples were amplified in triplicate using the following cycles: $95^{\circ} \mathrm{C}$ for $2 \mathrm{~min}, 35 \mathrm{cycles}$ of $95^{\circ} \mathrm{C}$ for $15 \mathrm{sec}, 60^{\circ} \mathrm{C}$ for $30 \mathrm{sec}$ and $72^{\circ} \mathrm{C}$ for $20 \mathrm{sec}$.

Cell migration and invasion assays. Cell migration assay was performed in Transwells (Corning Company). Cells in $0.5 \mathrm{ml}$ serum-free medium were placed in the upper chamber, whereas the lower chamber was loaded with $0.8 \mathrm{ml}$ medium containing $10 \%$ FBS. The total number of cells that migrated to the lower chamber were counted after incubation for $48 \mathrm{~h}$. Six optional visual fields were chosen for counting. The Transwell cell invasion assay was performed using Transwells that were pre-loaded with a layer of Matrigel (Sigma-Aldrich) on the upper surface. The rest of the experimental procedure was the same as with the cell migration assay.

Xenograft studies. All animal experiments were performed strictly in accordance with the related ethics regulations of our university. Ten six-week-old female nude mice were each injected with SW620-OCT4-shRNA (OCT4-knockdown cells) or SW620-mock-shRNA (mock control cells) $\left(5 \times 10^{6}\right)$ into the spleen. Mice were sacrificed 30 days after injection. The liver was embedded in paraffin and sectioned for H\&E staining to examine for metastasis.

Immunohistochemistry. Anti-human OCT4 antibody from Abcam was used for immunohistochemical (IHC) staining. Colorectal cancer tissue from our hospital was used. Tissues were formalin fixed, paraffin embedded (PPFE) slides. Each tissue was confirmed by experienced pathologists. Primary antibodies for OCT4 were diluted at 1:250 for IHC. Secondary antibody was used at a 1:200 dilution. The percentage of the positive cell population was categorized for scoring: $0,0 \%$ of the cell population was positive; $1,1-25 \%$ of the cell population was positive; $2,26-50 \%$ of the cell population was positive; $3,51-75 \%$ of the cell population was positive; $4,76-100 \%$ of the cell population was positive. The staining intensities were scored as: -, negative staining; + , weak staining intensity; ++ , medium staining intensity; +++, strong staining intensity.

Statistical analysis. The results are reported as means \pm SD. Statistical analysis was performed using the Student's t-test or Chi-square test as appropriate. $\mathrm{P}<0.05$ was considered to indicate a statistically significant difference.

\section{Results}

OCT4 knockdown inhibits cell migration and invasiveness in vitro. The protein levels of OCT4 were evaluated in the mock control and OCT4-knockdown cells by western blotting. As shown in Fig. 1A, OCT4 proteins were strongly expressed in the mock control cells. However, as expected, SW620-OCT4-shRNA cells demonstrated a decreased OCT4 protein expression level. We first assessed the effects of OCT4 expression on CRC cell migration and invasion with Transwell migration and invasion assays. OCT4-knockdown cells (SW620-OCT4-shRNA) exhibited a significant decrease in migration. The number of SW620-OCT4-shRNA cells migrating to the lower chamber was $\sim 6$ times lower than the number of migrating SW620 mock control cells (Fig. 1B). An in vitro cell invasion assay was performed based on the principle of the Boyden chamber assay, and Matrigel matrix was used as a reconstituted basement membrane in vitro. The number of cells migrating through the Matrigel matrix was counted and the result is presented in Fig. 1C. The OCT4knockdown cells showed significantly reduced invasiveness compared to the mock control cells $(\mathrm{P}<0.05)$. These data 
A

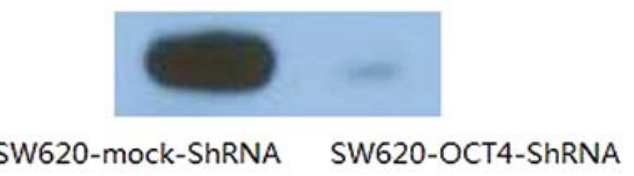

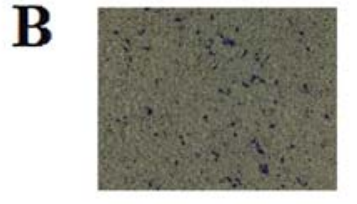

SW620-OCT4-ShRNA

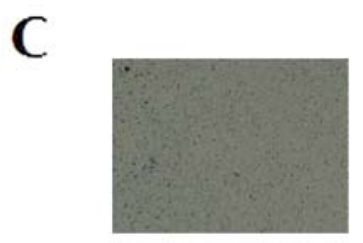

SW620-OCT4-ShRNA

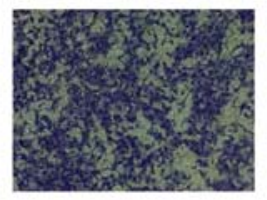

SW620-mock-ShRNA

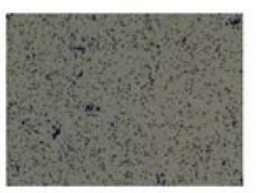

SW620-mock-ShRNA

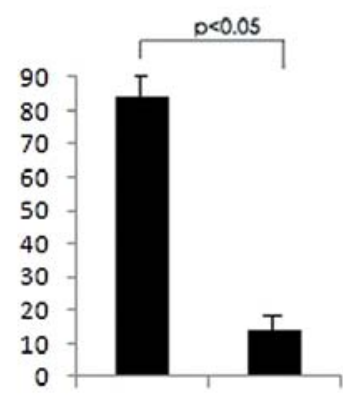

SW620-mock-ShRNA SW620-OCT4-ShRNA

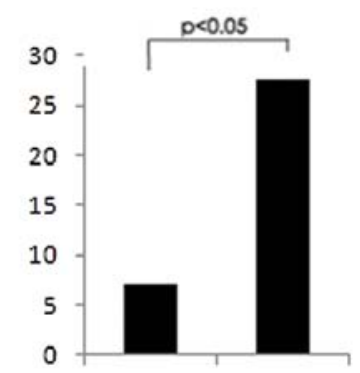

SW620-OCT4-ShRNA SW620-mock-ShRNA

Figure 1. OCT4 knockdown decreases cell migration and invasion of colorectal cancer cells. (A) OCT4 protein expression was determined in the SW620-mockshRNA and SW620-OCT4-shRNA CRC cells by western blot analysis. (B) Left panel: migration analysis of SW620-mock-shRNA and SW620-OCT4-shRNA cells using the Transwell migration assay. Right panel: quantification data (means \pm SD) of the cells that migrated to the lower chambers. (C) Left panel: the invasion analysis of SW620-mock-shRNA and SW620-OCT4-shRNA cells using a Transwell cell invasion assay. Right panel: the quantification results (means $\pm \mathrm{SD}$ ) of the cells that migrated to the lower chambers.
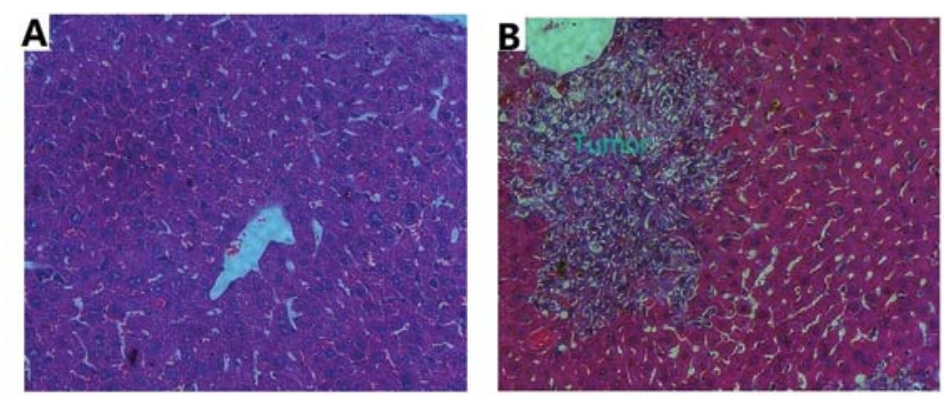

\section{C the impact of OCT4 expression on CRC liver metastasis in mouse model Group liver metastasis \\ SW620-OCT-4-ShRNA $3(30 \%)$ \\ SW620-MOCK-ShRNA $10(100 \%)$}

Figure 2. OCT4 knockdown decreases in vivo metastasis of colorectal cancer cells in mice. (A) H\&E staining of mouse liver tissue without metastasis. (B) H\&E staining of mouse liver tissue containing metastasis. (C) The average number of liver metastatic foci in the OCT4-knockdown group was significantly less than that in the mock-control group.

indicate that the inhibition of OCT4 expression in SW620 cells is associated with reduced invasive ability.

OCT4 knockdown reduces liver metastasis of SW620 cells in a mouse model. To investigate whether OCT4 silencing suppresses colorectal cancer liver metastasis, a hepatic metastasis mouse model was used. SW620-OCT4-shRNA and mock control cells were locally injected to the spleen, respectively. All of the mice subjected to tumor cell injection survived until sacrifice on Day 30. All of the mice in the mock-control group $(10 / 10,100 \%)$ demonstrated the formation of metastatic foci in the liver (Fig. 2B). The incidence of liver metastasis was significantly decreased in the OCT4 shRNA group $(3 / 10,30 \%)$ (Fig. 2A). Moreover, the average number of metastatic foci in the OCT4-knockdown group was significantly lower than that in the mock-control group (Fig. 2C). These data suggest that OCT4 knockdown inhibits the ability of colorectal cancer cells to form metastasis.

OCT4 induces epithelial-mesenchymal transition in SW620 cells. EMT is important for epithelial cancer metastasis. The characteristics of EMT include a switch from the expression of 
A

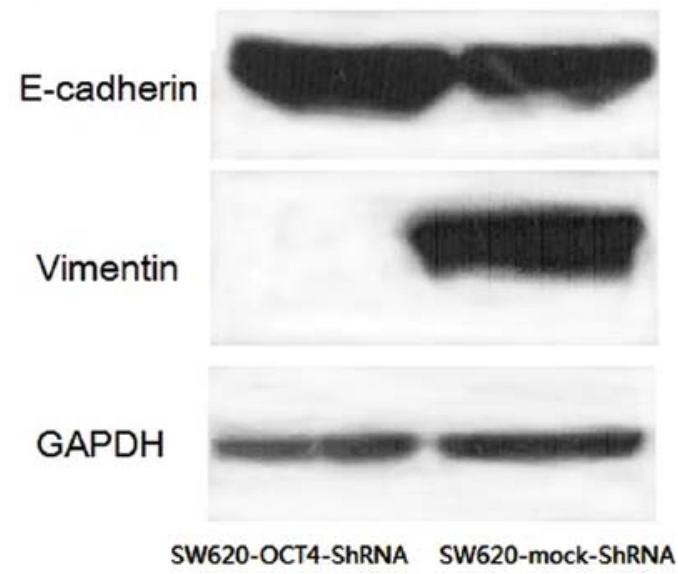

B E-cadherin
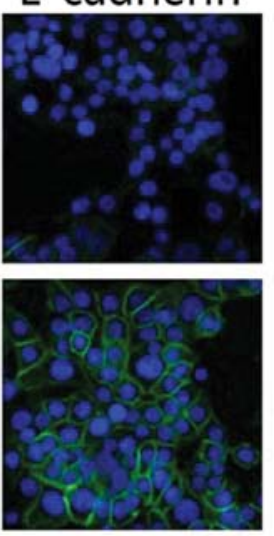

SW620-OCT4-ShRNA
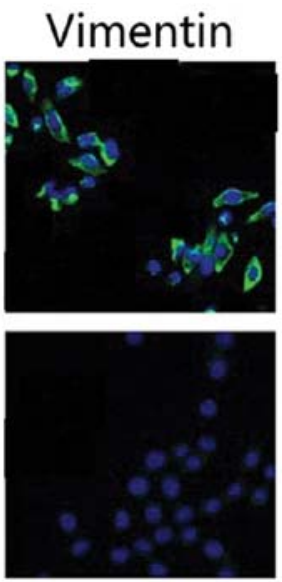

SW620-mock-ShRNA

Figure 3. OCT4 is involved in the EMT process in colorectal cancer cells. (A) Western blot analysis of the expression of E-cadherin and vimentin in SW620-mock-shRNA and SW620-OCT4-shRNA cells. (B) Immunofluorescence staining of SW620-mock-shRNA and SW620-OCT4-shRNA cells using anti-E-cadherin and vimentin antibodies.
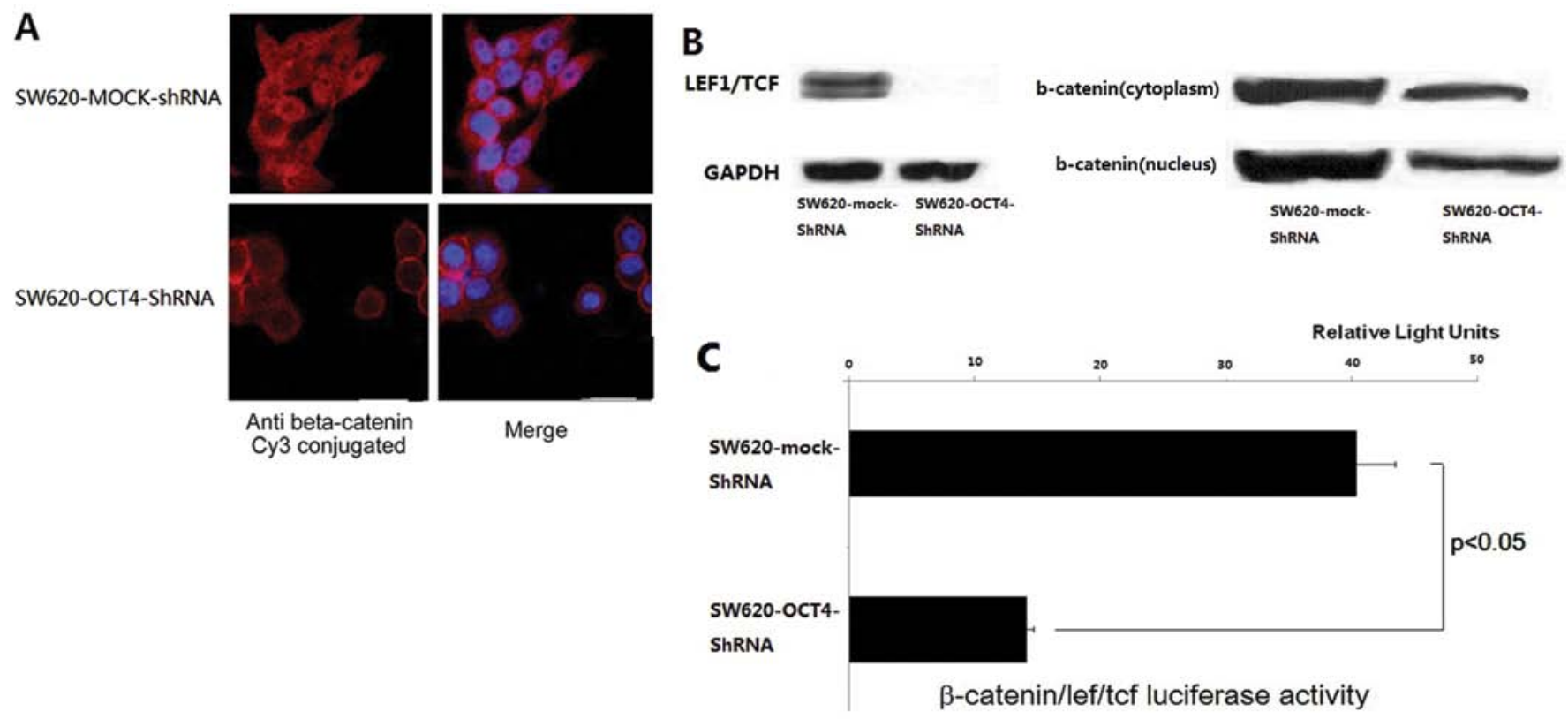

Figure 4. OCT4 is involved in the WNT signaling pathway in colorectal cancer cells. (A) Immunofluorescence images of SW620-mock-shRNA and SW620OCT4-shRNA cells stained with the anti- $\beta$-catenin antibody. (B) Western blot analysis of $\beta$-catenin expression using cytoplasmic and nuclear fractions of SW620-mock-shRNA and SW620-OCT4-shRNA cells. (C) LEF1/TCF1 luciferase activity in SW620-mock-shRNA and SW620-OCT4-shRNA cells.

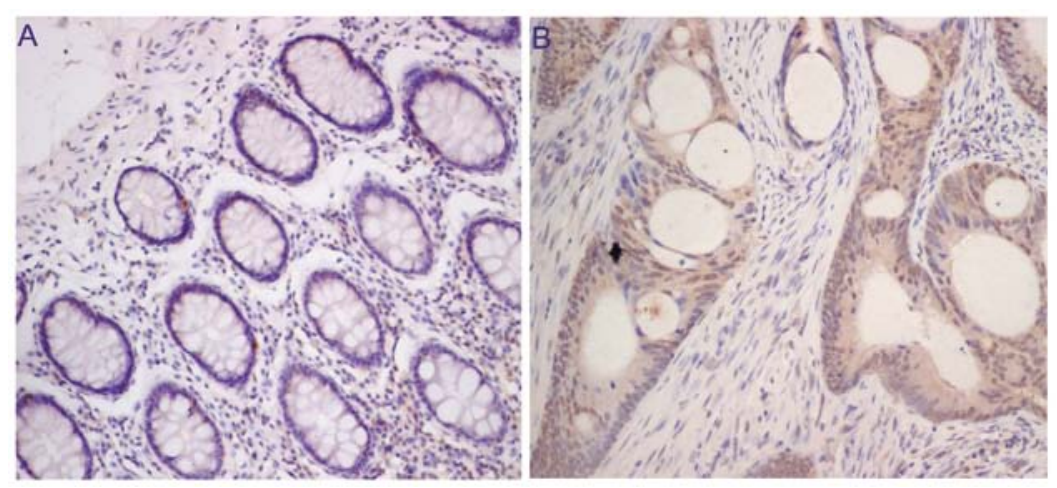

Figure 5. Immunohistochemical (IHC) staining of OCT4 in CRC tissues. (A) IHC image of OCT4-negative colorectal cancer tissue. (B) IHC image of OCT4positive colorectal cancer tissue. 
E-cadherin to vimentin and typical cell morphological changes. To examine whether knockdown of OCT4 alters the expression pattern of these two markers and cellular shape in SW620 cells, we used loss-of-function approaches in SW620 cells. We found that upon knockdown of OCT4, the level of E-cadherin was decreased whereas the level of vimentin was increased (Fig. 3A), consistent with altered staining patterns and intensities of these two markers (Fig. 3B). We also noted that the SW620-mockshRNA cells maintained mesenchymal cell morphology with spindle- and fibroblastoid-shape cells, while the SW620-OCT4shRNA cells showed epithelial cell morphology with epithelioid spreading cells (Fig. 3B). These results indicate that OCT4 is involved in the EMT process in colorectal cancer cells.

Knockdown of OCT4 leads to reduction in $\beta$-catenin/TCF/LEF signaling in SW620 cells. In colorectal cancer, EMT is companied by nuclear translocation of $\beta$-catenin and the activation of the WNT/ $\beta$-catenin signaling pathway (18-20). Following cell staining with the $\beta$-catenin antibody, we found that OCT4 knockdown induced the accumulation of $\beta$-catenin in the cell membrane and the reduction in cytoplasmic and nuclear $\beta$-catenin in SW620 cells compared to the mock-control (Fig. 4A). Western blot analysis further confirmed the results (Fig. 4B). $\beta$-catenin serves as an activator of T-cell factor (TCF) and activates transcription of downstream targets in the canonical WNT pathway (21). To evaluate the effect of OCT4 expression on the WNT pathway, LEF/TCF activity was assessed using an LEF/TCF reporter luciferase assay. We found that OCT4 knockdown led to an $\sim 3$-fold decrease in $\beta$-catenin/TCF/LEF signaling activity (Fig. 4C). OCT4 knockdown resulted in upregulated $\beta$-catenin membrane translocation and reduced canonical WNT pathway activities.

High expression of OCT4 is correlated with liver metastasis in human colorectal cancer. We next investigated the levels of OCT4 expression in human colorectal cancers using IHC methods and aimed to ascertain whether the OCT4 expression level in primary colorectal cancer is associated with tumor liver metastasis in CRC patients. OCT4 expression was examined in paraffin-embedded primary tumor tissues from $50 \mathrm{CRC}$ patients. We found that weak OCT4 expression was detected in every sample of normal colorectal tissue (Fig. 5A), and $46 \%$ of CRC cases (23/50) exhibited high expression of OCT4 (Fig. 5B). Among the CRC cases with high expression of OCT4, the percentage of liver metastasis was $82.6 \%$. However, in the cases with low expression, the percentage of liver metastasis was $22.0 \%$ (11/50 cases). The difference achieved statistical significance $(\mathrm{P}<0.05)$.

\section{Discussion}

OCT4, a member of the POU family, is an octamer motifbinding transcription factor. It is highly expressed in stem cells and has been considered as a key molecule which could induce somatic cell pluripotency. OCT4 is essential for maintaining undifferentiated and pluripotent populations of cells. Kim et al $(21,22)$ reported that OCT4 can directly reprogram adult mouse and human neural stem cells to induced pluripotent stem (iPS) cells. These studies showed that OCT4 plays an important role in conferring the stemness of cells.
In general, cancer cells are similar to early embryonic cells exhibiting the properties of immortality, undifferentiation and invasion (23). Thus, it is vital to study genes associated with embryogenesis and tumorigenesis. In recent years, there have been some reports on the regulated expression of OCT4 in several cancer types (12-16). Metastasis is the neoplastic process responsible for most deaths from cancer as primary tumors can usually be surgically removed. Liver metastasis is the main reason for death among colorectal cancer patients. However, the specific molecular changes in CRC cells that promote the metastatic process are largely unclear. Some recent studies suggest that OCT4 may be involved in the metastasis of CRC $(14,16)$. This prompted us to ascertain whether OCT4 has a relationship with CRC metastasis. In the present study, we investigated the role of OCT4 in metastasisrelated activities in human colorectal cancer and its effects on the EMT process and WNT pathway activity. To our knowledge, this is the first report to study the role of OCT4 in a human colon cancer cell line by inhibiting endogenous OCT4 expression using an RNA interference technique. Metastatic cells undergo loss of adhesion and enhanced motility and degradation of the basement membrane. Therefore, we detected these capabilities of OCT4 in CRC SW620 cells. Knockdown of OCT4 by specific shRNA inhibited SW620 cell migration and invasion in a Transwell-based assay. After showing that knockdown of OCT4 reduced cell migration and invasion in vitro, we conducted an in vivo analysis. We compared the abilities of the OCT4 knockdown and the mock control cells to form liver metastasis in a mouse model. We found that the incidence of liver metastasis and the average number of metastatic foci were significantly decreased in the OCT4 shRNA group. These data suggested that OCT4 knockdown reduces the ability of colorectal cancer cells to form metastasis.

Epithelial-mesenchymal transition (EMT) is an important process for epithelial cancer and is often activated during cancer invasion and metastasis. During EMT, cancer cells undergo morphological changes from an epithelial polarized morphology to a mesenchymal fibroblastoid morphology and achieve increased migratory and invasive capabilities. Our present research demonstrated that OCT4 silencing induced typical cell morphological changes and EMT marker expression alteration. These results indicate that OCT4 is involved in metastasis through the EMT process in colorectal cancer cells. The WNT pathway is widely regarded as the crucial pathway for colorectal carcinogenesis $(24,25)$ and regulates EMT process to promote cancer metastasis. $\beta$-catenin forms complexes in the adhesion complex at the plasma membrane and the signaling complex in the nucleus (26). We observed that OCT4 knockdown induced relocalization of $\beta$-catenin from the cytoplasm/nucleus to the membrane and reduced LEF/TCF activity. Finally, in human tissue samples, increased levels of OCT4 expression in primary cancer were significantly correlated with liver metastases.

Our research for the first time demonstrated a relation between OCT4 and the EMT process. OCT4 may be involved in metastasis of CRC in a Wnt/ $\beta$-catenin signaling-dependent manner. OCT4 expression in primary cancer could be used as a marker to predict liver metastasis in colorectal cancer patients. 


\section{References}

1. Shike M, Winawer SJ, Greenwald PH, Bloch A, Hill MJ and Swaroop SV: Primary prevention of colorectal cancer. The WHO Collaborating Center for the Prevention of Colorectal Cancer. Bull World Health Organ 68: 377-385, 1990.

2. Winawer SJ, Fletcher RH, Miller L, et al: Colorectal cancer screening: clinical guidelines and rationale. Gastroenterology 112: 594-642, 1997.

3. Takahashi K and Yamanaka S: Induction of pluripotent stem cells from mouse embryonic and adult fibroblast cultures by defined factors. Cell 126: 663-676, 2006.

4. Takahashi K, Tanabe K, Ohnuki M, Narita M, Ichisaka T, Tomoda $\mathrm{K}$ and Yamanaka S: Induction of pluripotent stem cells from adult human fibroblasts by defined factors. Cell 131: 861-872, 2007

5. Zhang Y, Zhang X, Wang X, Gan L, Yu G, Chen Y, Liu K, Li P, Pan J, Wang J and Qin S: Inhibition of LDH-A by lentivirusmediated small interfering RNA suppresses intestinal-type gastric cancer tumorigenicity through the downregulation of Oct4. Cancer Lett 32: 45-54, 2012.

6. Asadi MH, Mowla SJ, Fathi F, Aleyasin A, Asadzadeh J and Atlasi Y: OCT4B1, a novel spliced variant of OCT4, is highly expressed in gastric cancer and acts as an antiapoptotic factor. Int J Cancer 128: 2645-2652, 2011.

7. Chen Z, Xu WR, Qian H, Zhu W, Bu XF, Wang S, Yan YM, Mao F, Gu HB, Cao HL and Xu XJ: Oct4, a novel marker for human gastric cancer. J Surg Oncol 99: 414-419, 2009.

8. Kim RJ and Nam JS: OCT4 expression enhances features of cancer stem cells in a mouse model of breast cancer. Lab Anim Res 27: 147-152, 2011.

9. Iida H, Suzuki M, Goitsuka R and Ueno H: Hypoxia induces CD133 expression in human lung cancer cells by up-regulation of OCT3/4 and SOX2. Int J Oncol 40: 71-79, 2012.

10. Guo Y, Liu S, Wang P, Zhao S, Wang F, Bing L, Zhang Y, Ling EA, Gao J and Hao A: Expression profile of embryonic stem cell-associated genes Oct4, Sox2 and Nanog in human gliomas. Histopathology 59: 763-775, 2011.

11. Ikushima H, Todo T, Ino Y, Takahashi M, Saito N, Miyazawa K and Miyazono K: Glioma-initiating cells retain their tumorigenicity through integration of the Sox axis and Oct4 protein. J Biol Chem 286: 41434-41441, 2011.

12. Fang XF, Zhang WY, Zhao N, Yu W, Ding D, Hong X, Li LS, Zhang HR, Zheng S and Lin BY: Genome-wide analysis of OCT4 binding sites in glioblastoma cancer cells. J Zhejiang Univ Sci B 12: 812-819, 2011.

13. He W, Li K, Wang F, Qin YR and Fan QX: Expression of OCT4 in human esophageal squamous cell carcinoma is significantly associated with poorer prognosis. World J Gastroenterol 18 712-719, 2012.
14. Saigusa S, Tanaka K, Toiyama Y, Yokoe T, Okugawa Y, Ioue Y, Miki C and Kusunoki M: Correlation of CD133, OCT4, and SOX2 in rectal cancer and their association with distant recurrence after chemoradiotherapy. Ann Surg Oncol 16: 3488-3498, 2009.

15. Chang CJ, Chien Y, Lu KH, Chang SC, Chou YC, Huang CS, Chang $\mathrm{CH}$, Chen KH, Chang YL, Tseng LM, Song WS, Wang JJ, Lin JK, Huang PI and Lan YT: Oct4-related cytokine effects regulate tumorigenic properties of colorectal cancer cells. Biochem Biophys Res Commun 415: 245-251, 2011.

16. Gazouli M, Roubelakis MG, Theodoropoulos GE, Papailiou J, Vaiopoulou A, Pappa KI, Nikiteas N and Anagnou NP: OCT4 spliced variant OCT4B1 is expressed in human colorectal cancer. Mol Carcinog 51: 165-173, 2012.

17. Thiery JP: Epithelial-mesenchymal transitions in tumour progression. Nat Rev Cancer 2: 442-454, 2002.

18. Bates RC and Mercurio AM: The epithelial-mesenchymal transition (EMT) and colorectal cancer progression. Cancer Biol Ther 4: 365-370, 2005 .

19. Brabletz T, Hlubek F, Spaderna S, Schmalhofer O, Hiendlmeyer E, Jung $A$ and Kirchner T: Invasion and metastasis in colorectal cancer: epithelial-mesenchymal transition, mesenchymal-epithelial transition, stem cells and beta-catenin. Cells Tissues Organs 179: 56-65, 2005.

20. Floor S, van Staveren WC, Larsimont D, Dumont JE and Maenhaut C: Cancer cells in epithelial-to-mesenchymal transition and tumor-propagating-cancer stem cells: distinct, overlapping or same populations. Oncogene 30: 4609-4621, 2011.

21. Kim JB, Greber B, Arauzo-Bravo MJ, Meyer J, Park KI,Zaehres H and Scholer HR: Direct reprogramming of human neural stem cells by OCT4. Nature 461: 649-653, 2009.

22. Kim JB, Sebastiano V, Wu G, Arauzo-Bravo MJ, Sasse P, Gentile L, Ko K, Ruau D, Ehrich M, van den Boom D, et al: Oct4-induced pluripotency in adult neural stem cells. Cell 136: 411-419, 2009.

23. Monk M and Holding C: Human embryonic genes re-expressed in cancer cells. Oncogene 20: 8085-8091, 2001.

24. Gottardi CJ and Gumbiner BM: Adhesion signaling: how betacatenin interacts with its partners. Curr Biol 11: R792-R794, 2001.

25. Kim K, Lu Z and Hay ED: Direct evidence for a role of betacatenin/LEF-1 signaling pathway in induction of EMT. Cell Biol Int 26: 463-476, 2002.

26. Kolligs FT, Bommer G and Goke B: Wnt/beta-catenin/tcf signaling: a critical pathway in gastrointestinal tumorigenesis. Digestion 66: 131-144, 2002. 